\title{
Complex Monolayer Growth Dynamics of a Highly Symmetric Molecule: NTCDA on Ag(111)
}

\author{
Thomas Schmidt, ${ }^{*}, \ddagger \odot$ Helder Marchetto, ${ }^{\ddagger}$ Ullrich Groh, ${ }^{\dagger}$ Rainer H. Fink, ${ }^{\S}$ and Eberhard Umbach ${ }^{\dagger, \ddagger}$ \\ ${ }^{\dagger}$ Experimentelle Physik, Universität Würzburg, Am Hubland, 97074 Würzburg, Germany \\ ${ }^{\ddagger}$ Chemische Physik, Fritz-Haber-Institut der Max-Planck-Gesellschaft, 14195 Berlin, Germany \\ ${ }^{\S}$ Physikalische Chemie 2, Friedrich-Alexander-Universität Erlangen-Nürnberg (FAU), Egerlandstraße 3, 91058 Erlangen, Germany
}

\section{Supporting Information}

\begin{abstract}
The growth dynamics of the highly symmetric planar organic molecule NTCDA (1,4,5,6-naphthalene tetracarboxylic acid dianhydride) on $\operatorname{Ag}(111)$ is rather complex, already in the monolayer regime. This dynamics was investigated in situ with high lateral resolution using the aberration-corrected spectro-microscope SMART. Although the molecular structure of NTCDA is very similar to that of the slightly larger molecule PTCDA, the growth behavior of these two molecules is very different. Several differences of the static geometric, electronic, and vibrational structure have been described previously by several authors, but some dynamic
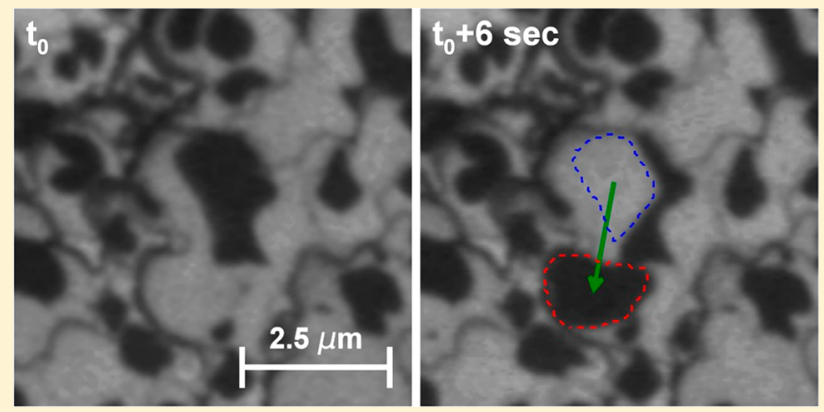
properties hardly accessible by other techniques could be observed in the present real time experiments using the SMART. For instance, it is found by direct observation that after decoration of steps and step bunches and depending on substrate temperature and surface morphology, a two-dimensional lattice gas of diffusing NTCDA molecules is formed. This increases in density upon continuous deposition of molecules before islands nucleate which subsequently grow in size while the density of the lattice gas first remains constant and then is reduced. Furthermore, in a certain temperature and coverage range some macroscopic islands (of micrometer size) abruptly change their shape and position on the time scale of few seconds. These "jumping 2-dim droplets" are observable for adsorption as well as for desorption. Moreover, previously observed 2-dimensional phase transitions can now be followed in real time, yielding further insight into an interesting but complex adsorption system.
\end{abstract}

\section{INTRODUCTION}

Organic thin films are of still increasing importance in various applications, for instance in organic photovoltaics, ${ }^{1,2}$ field-effect transistors, ${ }^{3}$ and, especially, light-emitting devices and displays. ${ }^{4,5}$ It is clear that the electric or optical properties of such organic films as well as their stability and processing properties strongly depend on the molecules, their size, shape, functional groups, intermolecular interaction, etc. It is also well established that the relative positions of the electronic levels at the interface between organic and inorganic material (e.g., metal contact), which are responsible for the charge injection, are related to the energetics or-in other words-to the electronic potentials of and chemical interaction between organic material and metal. ${ }^{6,7}$ However, it is less well-known, that many properties also decisively depend on the geometric structure and morphology of the organic thin films which can strongly vary depending on material, structure, and topology of the substrate, the interface formation, and the growth behavior of the organic film. Such properties can be electrical or exciton transport, e.g., exciton dispersion, ${ }^{8}$ optical quenching, ${ }^{9}$ and fluorescence yield. ${ }^{10}$ For instance, the fluorescence yield of a thin PTCDA film was found to vary by a factor of 20 depending on the substrate temperature and growth conditions accompanied by large changes in the optical spectra. ${ }^{10}$ Thus, for the optimization of the properties of organic devices and for the reproducibility of film preparation and results, it is of decisive importance to fundamentally understand the interplay between preparation and function, and especially between interface, thin film growth as well as geometric and electronic structure.

For this purpose we have recently published several papers which address the organic layer growth in detail, especially the interplay between substrate morphology, preparation parameters, formation of the interface, and growth of subsequent organic layers. ${ }^{11-17}$ In these publications we have concentrated on the system PTCDA on $\operatorname{Ag}(111)$ and its vicinal surfaces or on PTCDA on $\mathrm{Au}(111) .{ }^{18}$ We have discovered large-scale reconstructions, ${ }^{15-17}$ the formation of regular mesoscopic patterns, ${ }^{15}$ the occurrence of a wealth of molecular superstructures, ${ }^{17}$ different nucleation processes, ${ }^{12,13,18}$ the develop-

Special Issue: Hans-Joachim Freund and Joachim Sauer Festschrift

Received: August 31, 2018

Revised: December 18, 2018

Published: January 3, 2019 
ment and behavior of rotational and mirror domains for the first two layers, ${ }^{14,19}$ and the dependence of various growth mechanisms on preparation parameters and substrate morphology. ${ }^{12,13}$ Many of these observations strongly correlate with the chemisorptive bonding between PTCDA and the Ag substrate while the much weaker bonding on $\mathrm{Au}(111)$ resulted in significant differences. Of course, the question arises how much these observations depend on the specific molecule PTCDA. Thus, the next logical step is to investigate the differences that occur when a slightly different molecule with otherwise similar properties is utilized. For that purpose, we have chosen NTCDA (1,4,5,6-naphthalene tetracarboxylic acid dianhydride), which has the same symmetry and functional groups but a smaller aromatic core (naphthalene instead of perylene) and hence a smaller electrostatic quadrupole moment. We have again chosen $\operatorname{Ag}(111)$ as model substrate since much is known from investigations with conventional (static) surface analysis techniques. For NTCDA again, chemisorptive bonding, strongly modified interface states, and highly ordered superstructures are found on this substrate as for PTCDA (see below). The results and especially the dynamic adsorption behavior are very different for NTCDA as will be described in this work. In particular, some very interesting effects have been observed.

The monolayer regime of NTCDA on $\operatorname{Ag}(111)$ has been studied in quite some detail using conventional methods. From LEED, SPA-LEED, and STM studies it is known that NTCDA forms highly ordered monolayers on $\operatorname{Ag}(111), \operatorname{Ag}(110)$, and $\mathrm{Ag}(100)$ as well as on $\mathrm{Cu}(100) .{ }^{20}$ In most of these cases, two or more highly ordered monolayers are identified which occur in certain coverage and temperature regimes; especially on $\mathrm{Ag}(111)$ most authors discovered at least two modifications, a so-called "relaxed" monolayer ("r-ML") which is commensurate to the substrate and occurs for coverages below about $90 \%$ of saturation and a "compressed" monolayer ("c-ML") which has a point-on-line relation to the $\operatorname{Ag}(111)$ substrate and forms at saturation. ${ }^{20-24}$ The latter usually coexists with the r-MLstructure at coverages between 0.9 and 1.0 (where 1.0 is defined as saturation of flat lying monolayer molecules). By a careful SPA-LEED analysis Kilian et al. ${ }^{22}$ found a third, onedimensionally incommensurate monolayer state in the coverage range between 0.9 and 1.0 which coexists with the r-ML and c-ML monolayer states. NEXAFS investigations showed that all these monolayer states prepared at room temperature or above have an orientation with the molecular plane parallel to the substrate. ${ }^{25,26}$ However, Braatz et al. ${ }^{24}$ discovered a further modification by STM, IR, and SPA-LEED at $76 \mathrm{~K}$ which can be prepared by squeezing molecules from a second layer into empty spaces of the r-ML structure; this state is characterized by vertical NTCDA molecules bonding via the anhydride group and does not occur on the c-ML. Finally, for highly ordered monolayers ( $\mathrm{r}-\mathrm{ML}$ and $\mathrm{c}-\mathrm{ML}$ ) an activated, reversible order-disorder phase transition occurs upon cooling $^{27}$ which is accompanied by significant changes in the electronic structure; during this "unusual" two-dimensional (2dim) phase transition several further, highly ordered superstructures were observed which were not identified in detail (unpublished results and ref 28).

Model structures of geometric arrangement of the monolayer molecules were derived from LEED patterns and STM images which were usually, but not always, very similar to each other. ${ }^{20-28}$ While for the r-ML structure the findings and conclusions (6 domains, commensurate adsorption sites, 2 different molecules per unit cell, parallel azimuthal orientation ${ }^{20-24}$ ) essentially agree (apart from the orientation of the rows), there are differences between the various reports for the c-ML phase. While the older LEED and STM reports ${ }^{20-22}$ suggested molecular rows with the same azimuthal orientation within the rows but rotated with respect to adjacent rows (two molecules per unit cell with commensurate ${ }^{21}$ or incommensurate adsorption site $^{22}$ ), high resolution STM data recently found four molecules per unit cell where all neighboring molecules are azimuthally rotated with respect to each other. ${ }^{24}$ It is also important to report from a NIXSW study ${ }^{29}$ that at least for the r-ML state the molecules are adsorbed rather closely to the substrate and are drastically distorted as also observed for PTCDA/ $\operatorname{Ag}(111)$; this observation is consistent with a chemisorptive interaction with this substrate.

The latter conclusion could also be drawn from several investigations addressing the electronic and vibrational structure. NEXAFS measurements, ${ }^{25,26,30}$ photoemission experiments, ${ }^{25,26,31,32}$ laser studies (2PPE), ${ }^{33-36}$ and vibrational spectroscopies ${ }^{24,36-38}$ all clearly and consistently show that the molecules in the monolayer have a strong chemical interaction with the substrate which leads to strong shifts of the frontier orbitals, new interface states, very intense XPS satellites, and very different vibrational spectra as compared to isolated or weakly bound (physisorbed) molecules like those in the second molecular layer and above.

In several of these studies also the growth of the second and higher layers is investigated. It appears that the formation of these layers strongly depends on the preparation conditions and of the structure of the first layer. Thus, a detailed understanding of the formation of first layer may also clarify some of the open questions concerning the growth of higher layers.

\section{EXPERIMENTAL SECTION}

The instrument used for the present experiments is the SMART spectro-microscope ${ }^{39-41}$ installed at the UE49-PGM beamline of the BESSY-II storage ring of the HelmholtzCenter Berlin for Materials and Energy (HZB). SMART is equipped with an aberration corrector, compensating simultaneously both, chromatic and spherical aberrations, ${ }^{42-44}$ and with an aberration-corrected imaging energy filter. A lateral resolution of better than $2.6 \mathrm{~nm}$ was experimentally demonstrated in the low-energy electron microscopy (LEEM) mode. ${ }^{45,46}$ The design of the specimen chamber enables the in situ deposition of, e.g., organic material under grazing incidence $\left(20^{\circ}\right)$ onto the sample surface at the measurement position in front of the objective lens. The sample temperature is regulated by radiative and electronbombardment heating and monitored by a thermocouple. Thus, the growth or desorption of the NTCDA film could be directly observed in real time. For deposition a Knudsen cell type evaporator was used. The deposition rate was varied between 0.06 and $1.0 \mathrm{ML} / \mathrm{min}$ ( $\mathrm{ML}=$ monolayer coverage $)$ by adjusting the evaporator temperature between 403 and $438 \mathrm{~K}$. One ML corresponds to the deposited amount required to achieve a completely closed relaxed first NTCDA layer (of parallel oriented molecules) on the surface at $335 \mathrm{~K}$. At this temperature NTCDA desorption from the Ag substrate can be neglected. The base pressure of the measurement chamber was better than $3 \times 10^{-10}$ mbar. A potential influence of the instrumental setup on the growth has been carefully checked and could be excluded in the same way as reported for 

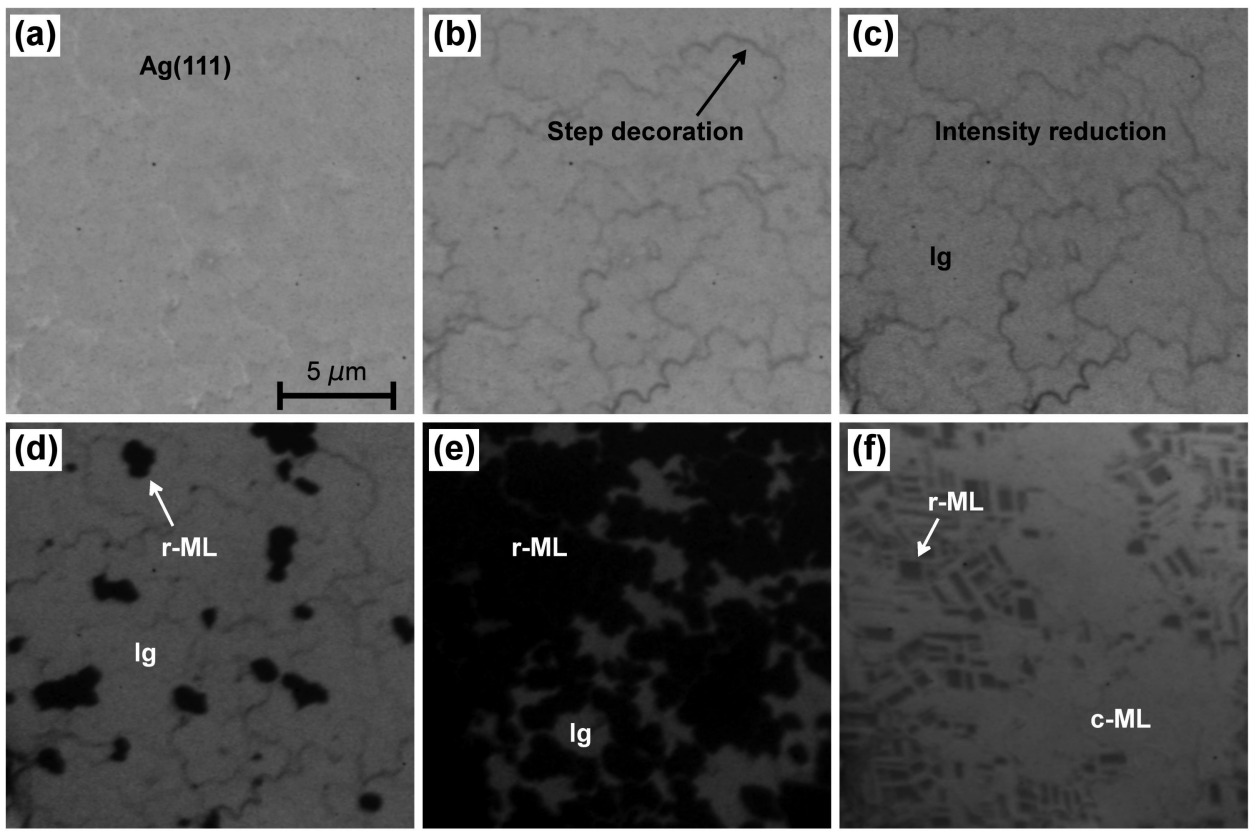

Figure 1. UV-PEEM images of the growth of NTCDA/Ag(111) at $350 \mathrm{~K}$. The deposition rate is $0.06 \mathrm{ML} / \mathrm{min}$. Nominal NTCDA coverages in the sequence $\mathrm{a}-\mathrm{f}$ are $0,0.03,0.09,0.25,0.92$, and $1.39 \mathrm{ML}$. The acquisition times are $4 \mathrm{~s}$ every $6 \mathrm{~s}$; the different phases during the growth are marked. The clean $\mathrm{Ag}(111)$ surface appears bright (a), while decorated steps at the initial growth are slightly darker (b). Whereas the r-ML islands are dark, the c-ML phase is bright (f). "lg" denotes the lattice gas covered $\mathrm{Ag}(111)$ surface. For comparison image scaling is kept constant for parts a-e, but for part $\mathrm{f}$, the intensity is increased by a factor 3.5 .

PTCDA. ${ }^{12}$ The kinetic energy of the electrons at the sample surface in the LEEM experiments was reduced below $5 \mathrm{eV}$ which is about the threshold at which no beam-induced damage is observed. The $\operatorname{Ag}(111)$ single crystal oriented within an accuracy better than $0.2^{\circ}$ has been cleaned by repeated cycles of Ar-sputtering $\left(600 \mathrm{eV}, 1 \mu \mathrm{A}, 5 \times 10^{-5} \mathrm{mbar}\right.$, $15 \mathrm{~min}$, room temperature) and subsequent annealing at $700-$ $800 \mathrm{~K}$ for about $15 \mathrm{~min}$. The cleaning progress was checked by PEEM, LEEM, LEED, and XPS.

In UV-PEEM mode, ultraviolet light from a $\mathrm{Hg}$ discharge lamp (maximum intensity at $h \nu=4.9 \mathrm{eV}$ ) releases electrons from the surface by photoemission. The emitted electrons are directly imaged on a two-dimensional detector and recorded over time by a CCD camera. The image contrast is determined by the local work function variations and by attenuation of the electrons emitted from the Ag substrate by deposited NTCDA molecules. In the NEXAFS-PEEM mode, monochromatic Xrays from the UE49-PGM undulator beamline at BESSY-II were used to directly image the emitted secondary electrons on the two-dimensional detector in real time. In this case the $\mathrm{X}$ ray polarization could be switched between s-polarization (with E-vector parallel to the surface) and p-polarization (with an E-vector component almost perpendicular to the surface) by shifting the magnetic poles of the undulator. ${ }^{47}$ In LEEM mode, electrons from an electron gun are decelerated near the surface, back-diffracted by the outermost surface layers and recorded as spatially resolved image. The contrast mechanism in this case is governed by the local surface reflectivity and by interference effects that occur upon electron reflection at different surface layers. ${ }^{48}$

\section{RESULTS AND INTERPRETATION}

3.1. Adsorption Behavior and Lattice Gas. In Figure 1, some selected UV-PEEM images are displayed which characterize the NTCDA adsorption at $350 \mathrm{~K}$ on the clean
$\mathrm{Ag}(111)$ surface. From temperature-programmed desorption (TPD) experiments, it is now known ${ }^{21,23,49}$ that above about $320 \mathrm{~K}$ the second and higher layers desorb whereas c-ML disappears between 350 and $400 \mathrm{~K}$. Above $400 \mathrm{~K}$, r-ML desorbs completely leaving a clean $\operatorname{Ag}(111)$ substrate (i.e., no dissociation occurs as for PTCDA on the same substrate). We hence expect that for $350 \mathrm{~K}$ at least $\mathrm{r}-\mathrm{ML}$ will adsorb.

Figure 1a shows the clean surface; there are no single steps, but step bunches are vaguely seen as weak features. Since the spatial resolution of UV-PEEM is $30 \mathrm{~nm}$, (uncovered) single steps, which are less than a nanometer wide and cause only minor work function contrast, are not detectable by UVPEEM. After deposition of $3 \%$ of r-ML (image b) the step bunches become visible in UV-PEEM because the initially deposited molecules diffuse over the surface until they reach a step or step bunch where they become trapped. There they induce a local work function increase which reduces the electron emission along step bunches (darker regions). In the (sub)monolayer regime, work function contrast is usually the dominating contrast mechanism in UV-PEEM but attenuation of the substrate photoemission by the adsorbate may also play a role (also leading to a reduction of emission). ${ }^{12}$ In the present case, we emphasize that the first $3 \%$ of the molecules arriving from the gas phase become immobilized (and visible) at steps or step bunches.

Upon further deposition of NTCDA first nothing but an overall "darkening" of the image (image c) happens; this darkening is proportional to the deposition time indicating that the NTCDA coverage continuously increases but no adsorbate islands are detected. This interesting observation is considered to be due to the occurrence of a two-dimensional gas phase ("lattice gas"; subsequently also denoted as "lg") and will be further elucidated below. After deposition of about $20 \%$ of a complete r-ML coverage, nucleation of islands sets in (dark areas in image $d$ ), which grow in size until the surface is nearly 

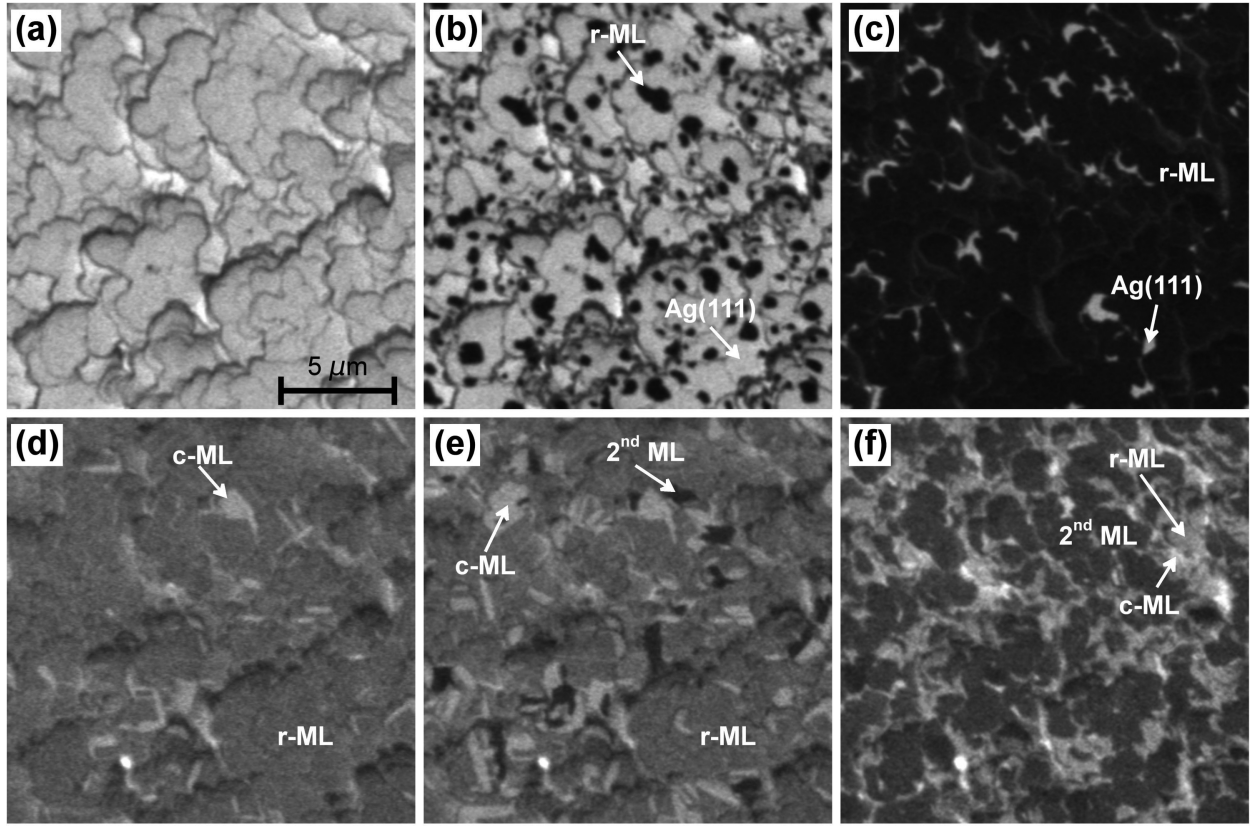

Figure 2. UV-PEEM image sequence of NTCDA growth on $\mathrm{Ag}(111)$ surface at $283 \mathrm{~K}$ and a deposition rate of $0.24 \mathrm{ML} / \mathrm{min}$. The nominal coverages are $0.0,0.26,0.93,1.19,1.26$, and $1.88 \mathrm{ML}$. The different phases and coverages are marked in the images and can be identified by their intensity. Intensity scalings in images $\mathrm{a}-\mathrm{c}$ and in images $\mathrm{d}-\mathrm{f}$ are constant, but the scaling is changed between image $\mathrm{c}$ and image $\mathrm{d}$.

completely covered by r-ML (image e). Further deposition then leads to the formation of islands of c-ML (lighter areas in image $\mathrm{f}$ ) but a completion of the c-ML phase could not be reached at this substrate temperature. From this observation and the fact that image $\mathrm{f}$ was obtained after deposition of 1.4 times more molecules than required for completing an $\mathrm{r}-\mathrm{ML}$ (1.1 should have been enough for reaching the saturated cphase $^{22,23}$ ), we conclude that the sticking coefficient reduces gradually to zero after completion of r-ML. This is consistent with the TPD/SPA-LEED data of Braatz et al., ${ }^{23}$ which demonstrated a stepwise disappearance of the compressed phase between 350 and $400 \mathrm{~K}$ upon heating a multilayer film.

Therefore, we performed experiments at lower temperature. In Figure 2 we show UV-PEEM data obtained from a sample kept at $283 \mathrm{~K}$. Image $2 \mathrm{a}$ represents a surface after step decoration (like Figure 1b). Further deposition of about 25\% of r-ML (Figure 2b) leads again to island nucleation (like in Figure 1d), but the islands are smaller and their density is higher. Moreover, the intensity of the island-free areas is only slightly reduced unlike the situation in Figure 1 (compare parts b and d). After deposition of $93 \%$ of r-ML (Figure $2 \mathrm{c}$ ), the islands have grown together leaving only small uncovered areas. Upon further deposition, islands of the c-ML phase nucleate and grow (bright areas in Figure 2d), but before these cover the entire surface, the second layer starts to grow (slightly darker areas in Figure 2e), and both types of islands (c-ML and second layer) grow simultaneously. Interestingly the second layer islands often grow on top of c-ML islands. Further deposition (amount: 188\% of r-ML; see Figure 2f) results in a complex situation with a nearly complete second layer (dark) while c-ML areas (bright) and r-ML areas (gray) are still seen between the second layer islands. The growth behavior for both temperatures with many more details is better seen in the movies available in the Supporting Information.

By comparing both series of images obtained at two different temperatures we note four major differences: (1) the second layer is only observed at the lower substrate temperature, and (2) also the compressed layer can only be completed at the lower temperature. Both (expected) observations are completely consistent with the TPD results as mentioned above. ${ }^{23}$ Also the third observation, the occurrence of smaller (and more) islands at lower temperature, is completely in accordance with nucleation theory ${ }^{50,51}$ and previous results. ${ }^{12,13,18,52}$

The most interesting observation is the fact that the onset of nucleation of the first layer islands ( $r-M L$ phase) is the more delayed the higher the substrate temperature is. This is accompanied by a continuous reduction of the substrate emission ("darkening"). We can exclude that the origin of this delay is a reduced initial sticking coefficient since the amount of evaporated molecules required to achieve a saturated $\mathrm{r}-\mathrm{ML}$ is about the same in both cases. We suggest that in this coverage regime a highly mobile, 2-dimensional NTCDA layer exists which can be described as 2-dim gas phase, often referred to as lattice gas. Upon further deposition its density increases leading to a reduction of photoemission from the substrate until a critical density is reached at which solid islands nucleate. These are visible in UV-PEEM as dark areas due to local work function enhancement and attenuation of substrate photoemission. The solid islands and the lattice gas are likely in equilibrium by (2-dim) adsorption and desorption processes occurring at the rims of the islands. Desorption from the surface into the (3-dim) vacuum is unlikely since there is sufficient bonding to the substrate even for the mobile molecules, as proven by the fact that the coverage remains constant when the shutter of the NTCDA evaporator is closed.

Of course, more experiments have been performed using different substrate temperatures $(265-365 \mathrm{~K})$ and deposition rates $\left((1-18) \times 10^{-3} \mathrm{ML} / \mathrm{s}\right.$, where $\mathrm{ML}$ refers to the complete $\mathrm{r}-\mathrm{ML})$. Their evaluation is summarized in Figure 3. In this figure, four phases are identified as a function of substrate temperature and amount of deposited molecules (which is related to the overall coverage for the r-ML phase): lattice gas 


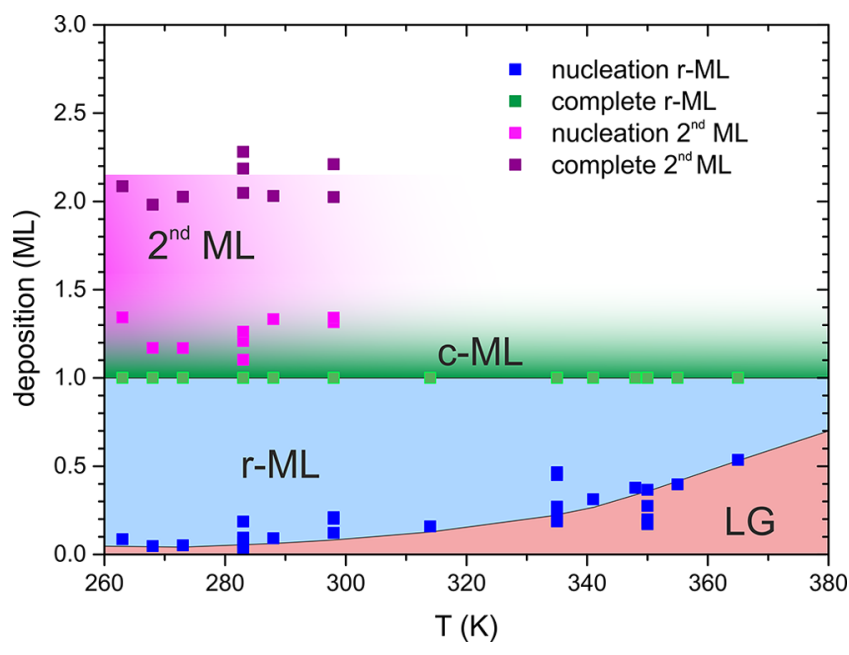

Figure 3. Overview about growth of the first layers. The deposition of $1 \mathrm{ML}$ is defined as the deposition time for the closure of the r-ML. Growth rates were between 0.06 and $1 \mathrm{ML} / \mathrm{min}$, hence the various squares belong to different growth rates (see Figure 5). Four growth regimes are marked: (i) lattice gas (LG) in red, (ii) r-ML (blue), (iii) c-ML (green), and (iv) 2nd ML (purple).

(LG; gray), r-ML, c-ML, and second layer. It is clearly seen that the coverage of the lattice gas strongly increases for higher temperatures and reaches even about $50 \%$ for $365 \mathrm{~K}$. This means that in this case the mobile state on the surface is filled by deposited (mobile) molecules up to a coverage of half a monolayer before nucleation of solid islands starts; then the density of the lattice gas remains at first constant while the islands grow upon further deposition. To our knowledge the direct observation of a lattice gas and its dependence on temperature is novel and was hitherto hardly possible. Also the observation that this 2-dim state can contain up to $50 \%$ of saturation coverage of a monolayer is surprising. We note, however, that numerous theoretical and experimental investigations have assumed or indirectly concluded the existence of a lattice gas (see below).

Figure 3 also gives the parameters for the observation of c$\mathrm{ML}$ which starts toward the end of the completion of r-ML but remains incomplete for temperatures above $300 \mathrm{~K}$. The formation of the c-ML occurs in coexistence with that of the second layer which is completed for temperatures below room temperature yielding a coverage of 2.2 referred to the r-ML. The average coverage of $\mathrm{c}-\mathrm{ML}$ (here $\sim 1.2$, referred to $\mathrm{r}-\mathrm{ML}$ ) is slightly higher than that given in the literature $\left(1.11^{22}\right)$ which could be related to the present calibration because r-MLs that were not annealed were here considered as "complete". The details of the subsequent filling of these four phases are much more complicated than Figure 3 suggests. This can be derived from Figure 2 and from the following figures as well as by careful inspection of the movies given in the Supporting Information.

3.2. Quantitative Evaluation of the Growth Behavior of the First Two Layers. The advantage of the present UVPEEM experiments is that each of the phases is clearly distinguishable from the others due to their different local work function and attenuation such that the photoemission signals and hence the contrasts are different (see, e.g., Figure 2 ). On the basis of this information, we extracted the intensities and areas of the different phases from each image of a deposition sequence. Thus, one can follow the development of the different phases as a function of time. The elapsed time is directly related to the exposure (number of deposited molecules) by a constant deposition rate.
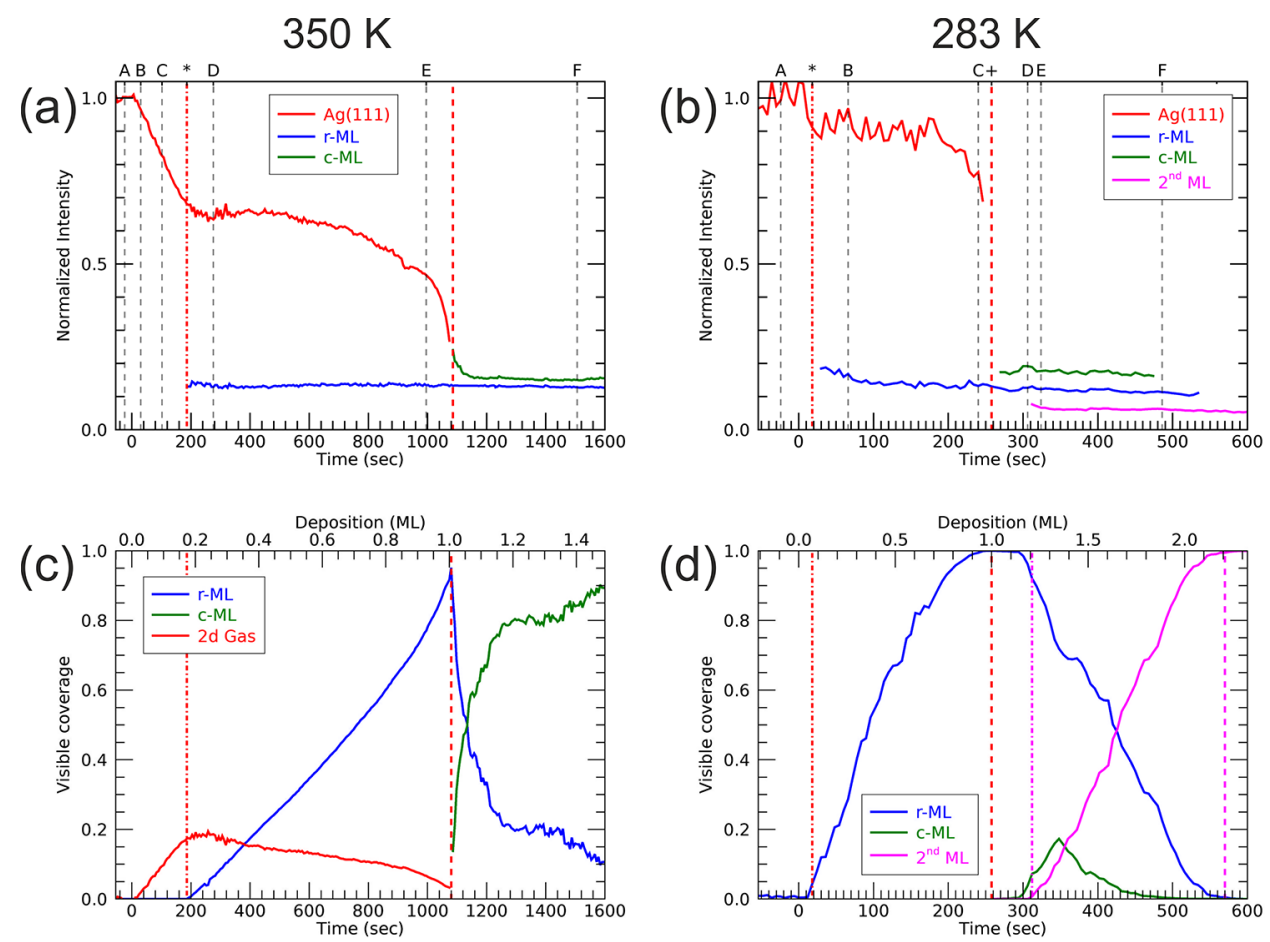

Figure 4. Temporal behavior of NTCDA growth on $\mathrm{Ag}(111)$ for high $(350 \mathrm{~K})$ and low $(283 \mathrm{~K})$ temperature, left and right column, as shown in Figures 1 and 2, respectively. Analysis of the local image intensity (top row) and the visible coverages (bottom row) for the lattice gas (red), r-ML (blue), c-ML (green), and 2nd ML (purple). 
Results of such evaluations are shown in Figure 4. Figure 4a displays the development of the intensity per unit area as a function of time (i.e., exposure) at $350 \mathrm{~K}$ for the three "phases": $\operatorname{Ag}(111)$ areas (with 2-dim NTCDA gas), relaxed NTCDA (r-ML), and compressed NTCDA (c-ML). The data were derived from consecutive images (frames) of a movie such as those of Figure 1. In Figure 4c, these intensities are converted into total coverages ( $y$-axis) by multiplying the evaluated (total) areas within the measured frame with the concentration of molecules within the respective phase. The concentration of the 2-dim gas phase is derived from the reduction of the Ag intensity per unit area as a function of dose assuming a sticking coefficient of 1 for coverages in the range up to a complete monolayer of the relaxed phase. This assumption is consistent with all our measurements at various temperatures.

In Figure $4 \mathrm{a}$, we notice that the $\mathrm{Ag}$ intensity after a very short delay (decoration of steps) is steadily reduced indicating an increase of coverage in the 2-dim gas phase, until after about $190 \mathrm{~s}$ of dosing (vertical line with an asterisk in Figure 4a) the nucleation of the r-phase sets in (blue line) which is equivalent to the appearance of dark areas (see Figure 1d). These areas grow in number and size but their coverage (emission intensity) remains constant. With the start of nucleation the intensity reduction of the Ag signal (i.e., coverage increase of the 2-dim gas phase) first levels off and then decreases again (Figure $4 c$, red curve). This decrease of coverage or density of the 2-dim gas phase is accompanied by an acceleration of the growth of the r-phase (the slope of the blue curve in Figure $4 \mathrm{c}$ is steeper than unity; see upper $x$-axis) indicating that the equilibrium between adsorbing and desorbing molecules at the rims of the r-phase islands from and to the 2-dim gas phase changes with number and size of the islands. This process is accelerated after $800 \mathrm{~s}$ of deposition (equivalent to a coverage of $\sim 80 \%$ of the r-phase) as can be derived from the slope of the blue curve in Figure 4c. The decrease of the red curve in Figure 4c (coverage of 2-dim gas phase) after $900 \mathrm{~s}$ is consistent with the enhanced growth of the r-phase islands.

The r-phase is not yet fully closed when the formation of the c-phase starts. It is clearly seen in the movie and indicated by the blue and green curves of Figure $4 c$ that the formation of the c-phase starts at areas between r-phase islands; i.e., it starts by condensation from the 2-dim gas phase. Immediately after the first nuclei have been formed, the c-phase then extends converting the r-phase into the c-phase by squeezing additional molecules into the r-ML. This process levels off after $200 \mathrm{~s}$ and continues only very slowly beyond $80 \%$ coverage of the cphase at this temperature. Apparently, the sticking coefficient drops to very small values beyond this point and eventually to zero.

The evaluation of an adsorption experiment performed at a substrate temperature of $283 \mathrm{~K}$ (see also Figure 2) is displayed in Figures 4, parts b and d. In this case, the deposition rate was more than four times higher (compare $x$-axes). There are significant differences to the adsorption at $350 \mathrm{~K}$. First of all the second and higher layers are stable and hence appear after doses above a monolayer of NTCDA (pink curves). Second, the coverage of the 2-dim gas phase remains much lower, and hence, nucleation of islands of the r-phase starts at much lower doses. In fact, there is also a decrease of the substrate signal after the shutter of the evaporator is opened and before the formation of the r-phase sets in (vertical line, marked by asterisks), but this is much smaller (and noisier for experimental reasons). Third, the growth of the r-phase is reduced beyond $80 \%$ of an r-phase monolayer and reaches a plateau between 240 and $300 \mathrm{~s}$ without anything else happening. Then the formation of the c-phase starts (green curve) and shortly after the growth of the second layer (pink curve). Since the c-phase forms from the r-phase by squeezing in $20 \%$ more molecules, the coverage of the r-phase is reduced at the same speed as that of the c-phase is increased. However, the formation of the c-phase can only be observed for a few seconds because the second layer nucleates on top of the cphase, which is hence no longer distinguishable by PEEM. The second layer is completed after $570 \mathrm{~s}$ as indicated by a vertical line in Figure 4d.

It remains to be understood what is happening between 240 and $300 \mathrm{~s}$. We assume that in this range a 2-dim gas phase is filled which diffuses on top of the r-phase but is not distinguishable from the latter because this already appears dark. Once a certain density is reached this 2-dim gas phase provides the additional molecules for the c-phase and the molecules for the second layer. We speculate that the formation is kinetically hindered and hence occurs with some delay because the squeezing in of molecules into a highly ordered monolayer ( $r$-phase) requires rearrangement of adsorbed molecules on a large scale and hence may occur as activated process. Moreover, the second layer predominantly adsorbs on c-phase domains; i.e., it apparently prefers the more densely packed c-phase as substrate, probably because of an enhanced van-der Waals interaction between second layer and c-phase.

When we compared the occurrence of the 2-dim gas phase ("lattice gas") on the Ag surface for different experiments (red curves in Figure 4) we noticed a significant temperature dependence. Thus, we plotted the maximum coverages of this lattice gas before nucleation of the r-phase vs the inverse temperature (Arrhenius plot). The result is displayed in Figure 5. This figure contains data points from different experiments with significantly varying deposition rates which were recorded on very different surface areas. Nevertheless they roughly follow a straight line as indicated yielding an "activation energy" of $0.26 \mathrm{eV}$. The possible meaning of an activation barrier is discussed below.

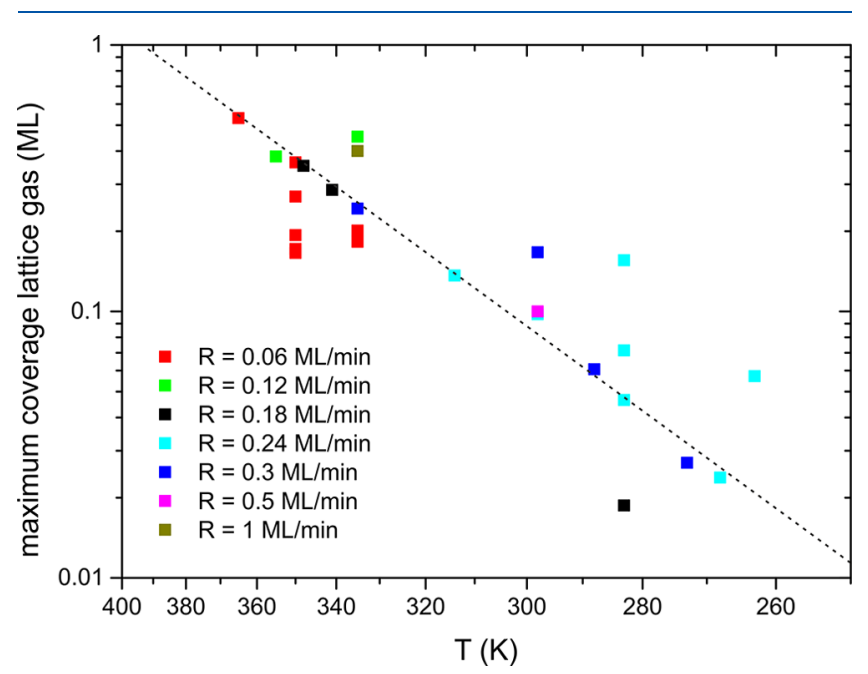

Figure 5. Temperature dependence of the maximum coverage of lattice gas for different deposition rates. The slope of the line in the Arrhenius plot corresponds to an activation energy of $0.26 \mathrm{eV}$. 

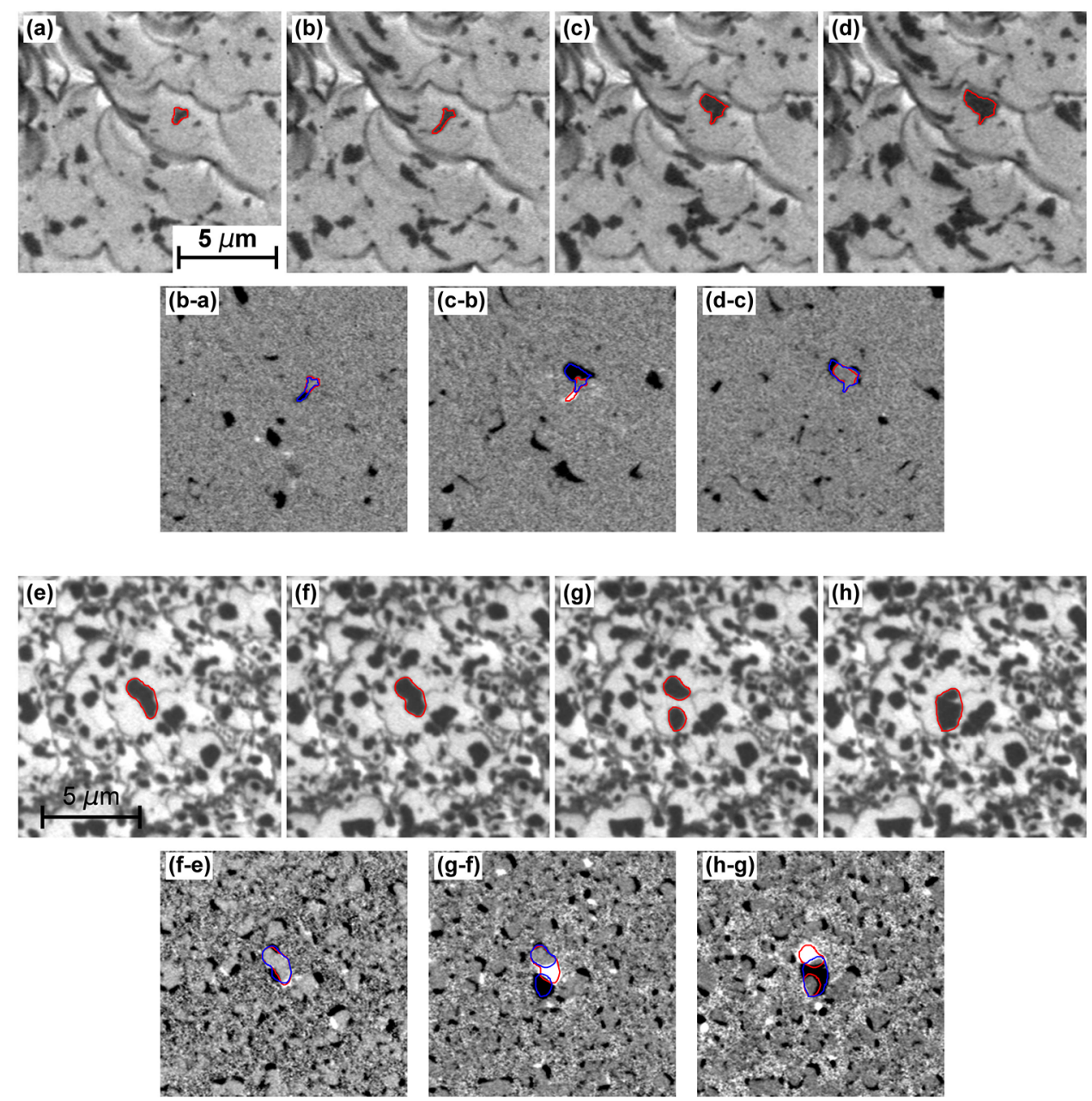

Figure 6. Mesoscopic mobility of NTCDA r-ML islands during growth at 350 (top) and $283 \mathrm{~K}$ (bottom). Subsequent images were taken every 6 s. The differential images are added below and exhibit the movement of islands: gray, dark, and white mean no change, growth, and shrinking/ vanishing, respectively.
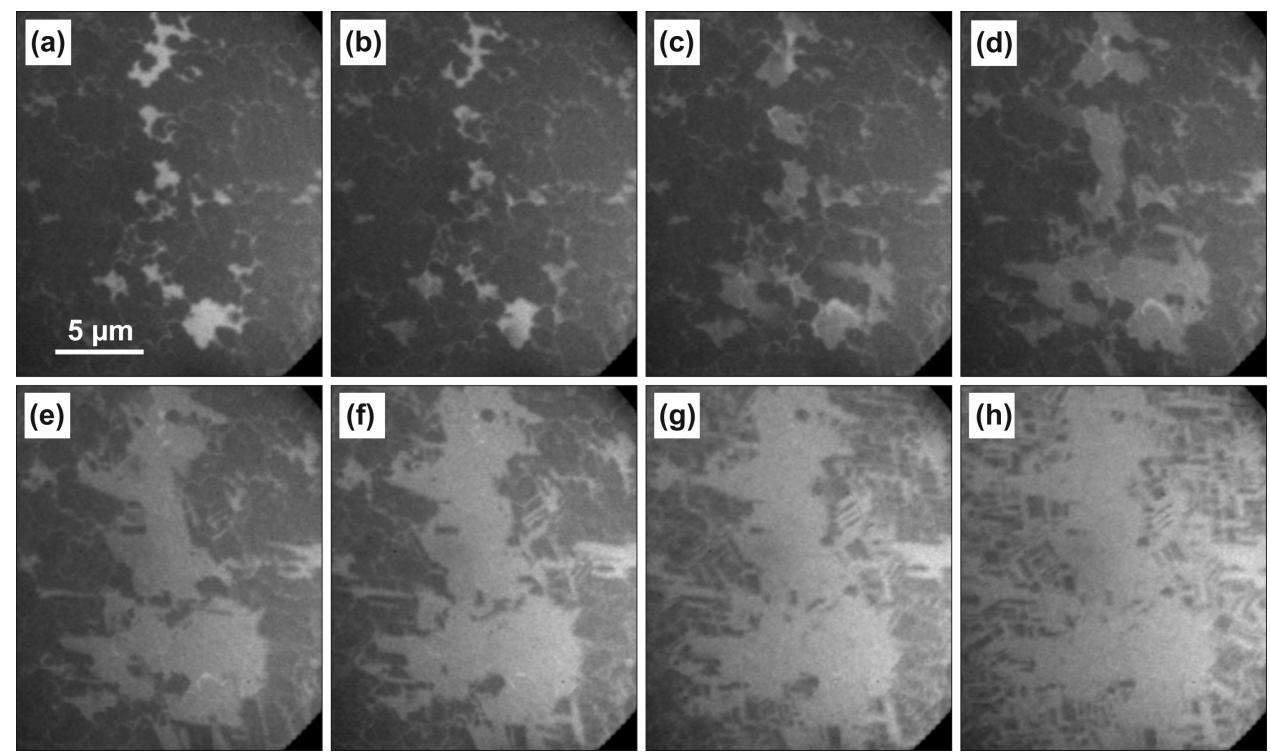

Figure 7. Formation of c-ML at $350 \mathrm{~K}$ and $R=0.055 \mathrm{ML} / \mathrm{min}$. The nominal coverages are (a) 0.990, (b) 0.995, (c) 1.0, (d) 1.005, (e) 1.01, (f) $1.02,(\mathrm{~g}) 1.055$, and $(\mathrm{h}) 1.17 \mathrm{ML}$.

3.3. Jumping Islands. In the course of our NTCDA experiments for adsorption as well as for desorption, we often observed large fluctuations of the shapes of the growing or vanishing islands occurring on the time scale of seconds. Since 

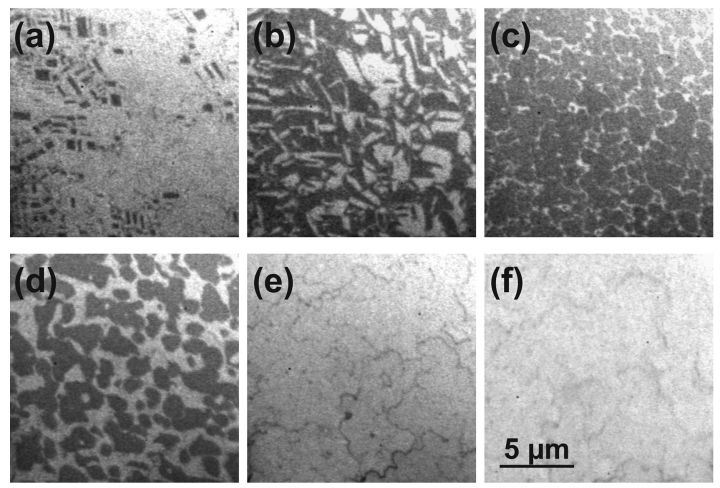

(e)

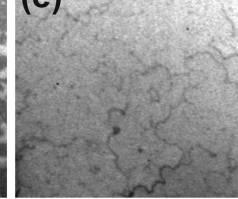

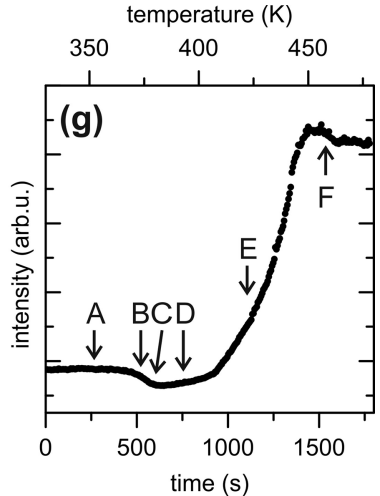

Figure 8. Desorption of nominal 1.2 ML NTCDA on $\operatorname{Ag}(111)$ as measured by UV-PEEM. The snapshots (a-f) show the heating sequence. Figure $8 \mathrm{a}$ is equivalent to Figure $7 \mathrm{~h}$. Different phases are observed during the desorption, appearing with different brightness in the PEEM images: c-ML is bright (see a), r-ML dark (c), lattice gas covered $\mathrm{Ag}(111)$ is bright ( $\mathrm{d}$ and e), and the clean $\mathrm{Ag}(111)$ surface is very bright (f). Step bunches decorated with NTCDA appear as dark lines (e). The image intensity of the entire experiment is plotted $(\mathrm{g})$ with markings A-F, where the snapshots a-f were taken.

we believe that this coordinated motion of about a million of chemisorbed molecules over distances of a micron is rather exceptional, we have extracted two examples from the movies which are displayed in Figure 6. Many more examples can be found in the movies in the Supporting Information.

The upper row shows four subsequent images of a movie recorded at $350 \mathrm{~K}$; the images were taken $6 \mathrm{~s}$ after one another equivalent with the adsorption of some additional molecules (about $2 \%$ of an r-ML). One can clearly see that the downward tip of the large (dark) r-phase island in Figure $6 \mathrm{~b}$ "jumps" to an area above; the difference image $(c-b)$ reveals this in more detail: the white spot represents the area where molecules disappeared whereas the black spot represents the area where new molecules appeared. There are several other spots that are black in the difference images (and some white), i.e. additional molecules were adsorbed or molecules disappeared. Note that the average distance of this motion of about a million molecules within $6 \mathrm{~s}$ is $1 \mu \mathrm{m}$.

Another example is shown in the third row (also four subsequent images); the differences between these images are displayed in the fourth row. These images were recorded at a different area on the $\mathrm{Ag}$ sample at a lower temperature (283 $\mathrm{K})$. Here, one can clearly observe an island in the middle (marked by red line) that first grows and then splits into two parts, which merge again in the next image at a slightly different area. The difference images reveal that indeed in the island in the center the largest change occurs but that in other areas also significant changes happen which can also be attributed to "jumping islands".

3.4. Formation of the Compressed Phase. Next we consider the dynamic process of formation of the compressed phase from the relaxed phase in more detail. Also this process occurs on large scales involving the simultaneous, collective motion of millions of molecules. Figure 7 displays some selected images from an adsorption movie that was recorded at $350 \mathrm{~K}$ at which the transformation process is best seen because no second layer can condense on the monolayer covering the processes that occur underneath.

In Figure $7 \mathrm{a}$, which has been recorded $1074 \mathrm{~s}$ after the start of the deposition, the r-ML (dark) covers already about $90 \%$ of the $\operatorname{Ag}(111)$ surface. The remaining holes with sizes in the $\mu \mathrm{m}$ range show emission from the $\mathrm{Ag}$ substrate attenuated by a high coverage $(\sim 50 \%)$ of lattice gas. The next image shown in
Figure $7 \mathrm{~b}$, taken $6 \mathrm{~s}$ after that of Figure $7 \mathrm{a}$, indicates that instead of completing the r-phase and closing the dark area some of the holes show the nucleation of the compressed phase; this is brighter than the r-phase but slightly darker than the lattice gas and hence well distinguishable. In the next image (Figure $7 \mathrm{c}$, taken again $6 \mathrm{~s}$ later), the c-phase has filled all former holes and starts extending into the r-phase areas. This process is continued in Figure 7d (again recorded $6 \mathrm{~s}$ later), which shows the rapid conversion of the r-phase into the cphase mainly around the former hole positions. In the next two images (Figure 7, parts e and f, recorded 6 and $18 \mathrm{~s}$ later), the conversion process slows down, probably because the sticking coefficient is reduced in this temperature and coverage range. In the further conversion process, elongated, needle-like cphase structures develop. These (flat) needles within one domain are perpendicular to each other; needles from different domains have angles of $30^{\circ}$ and $60^{\circ}$ relative to each other reflecting the symmetry of the (111) fcc substrate surface. Near saturation (Figure7, parts $g$ and $h$ ) the network of needles grows together until at saturation (not shown) the surface is covered by a continuous (gray) layer of c-phase molecules.

3.5. Additional Experiments. The versatile SMART instrument allows several other experiments, some of which have been performed in the course of the present investigation. Since these yielded important additional information, which need not have to be underlined by figures here, we abstain from showing such figures with explicit data but briefly report on the results. These corroborate the findings discussed above.

LEEM Experiments. In low energy electron microscopy, the $r$ - and the c-phase could not be distinguished, and hence, LEEM was less well suited to follow the adsorption behavior of the first two layers. The reason for this difference between LEEM and PEEM is that in PEEM the slightly different chemical bonding of these two states induces a different local work function which changes the emission from the substrate. In contrast, in LEEM, the back diffraction from both layers is apparently very similar, probably because the average vertical distance of the various atoms in the molecule from those of the substrate underneath is very similar in both phases. Thus, in LEEM, the different phases could not be distinguished. However, the step decoration at the start of the deposition could very clearly be followed, also for single atomic steps, not 
seen by PEEM. All other LEEM observations were consistent with those by PEEM.

Spatially Resolved NEXAFS Experiments. NEXAFS can be utilized to determine the orientation and the chemical bonding of molecules with respect to the substrate surface. ${ }^{25,53-60}$ With SMART, such experiments were done with sub-micrometer resolution. They confirmed the information from previous integral measurements ${ }^{25,56}$ also in mesoscopic dimensions: in both monolayer phases the molecules are adsorbed with the molecular plane essentially parallel to the substrate surface (accuracy of the present measurement: $\pm 20^{\circ}$ ). The same holds for the second layer although in this case the accuracy was only $\pm 40^{\circ}$ because of the simultaneous presence of the first layer.

Spatially Resolved LEED Experiments. LEED measurements with a LEED spot size of less than $1 \mu \mathrm{m}$ have been performed, for instance, on the c-phase as well as on 3-dim islands. The latter measurements will be reported in a forthcoming paper, while the monolayer pattern looked identical to those published for the c-phase before ${ }^{22}$ and hence are not shown. An important difference was that in the present experiment only one domain could be inspected by the $\mu$-spot LEED, and hence, only the pattern of one of the six rotational/reflection domains was seen. This proves that the islands observed indeed consist of large or even single domains or, in other words, that single domains have the size of several micrometers. We did not perform an analysis of the various monolayer domains as for PTCDA on $\operatorname{Ag}(111)^{14}$ but derive from our present observations that the analysis would have led to very similar results also in the NTCDA case.

Desorption Experiments. Of course, we also monitored the desorption behavior for NTCDA on $\operatorname{Ag}(111)$ starting with a compressed layer as well as starting with multilayers. While in the latter case interesting phenomena occur, which will be discussed in a forthcoming paper, the desorption behavior of the first layer is essentially the reverse of the adsorption behavior: first the additional molecules that make up the cphase desorb (Figure 8a), thus turning the c-phase layer into a complete r-phase layer (Figure $8 \mathrm{c}$ ). Upon further heating, this layer fractures, and the remaining r-phase islands shrink (Figure 8d) until they are completely resolved (Figure $8 \mathrm{e}$ ). Finally the uniform surface emission further increases (see graph in Figure 8g), indicating the gradual desorption of the 2dim gas phase, until at the end of the desorption process the dark step bunches become brighter when the decorating NTCDA molecules also desorb (Figure 8f). Thus, the desorption process mirrors the adsorption process including the lattice gas and step decoration.

Radiation Damage. Radiation damage either by UV (UVPEEM) or X-ray photons ( $\mu$-NEXAFS) or by electrons (LEEM) has been carefully checked for all experiments. While generally monolayers (and often also the second layer) are less sensitive to radiation damage because excitations within the adsorbate quickly delocalize into the (metallic) substrate before bonds are broken, multilayers or adsorbed microcrystallites may be modified (usually dissociated) by radiation. In the present case, the investigated mono- and bilayers were sufficiently stable on the time scales of the experiments as proven by comparing experiments with and without radiation under otherwise identical conditions. One exception is the stability of the second layer or microcrystallites (not discussed here) under UV radiation above room temperature $(330-350 \mathrm{~K})$. In this temperature range, we observed adsorption beyond the monolayer without UV lamp, while under operation of the UV lamp these layers desorbed (photon-stimulated or thermally activated) but without damage of the remaining monolayer.

\section{CONCLUDING DISCUSSION}

The adsorption system NTCDA on $\operatorname{Ag}(111)$ is more difficult to understand than it seems at first glance. This is also opposite to the initial intention of our investigation that the adsorption and layer growth of large organic molecules can be better understood by comparing a well-established prototype system, namely PTCDA on $\operatorname{Ag}(111)$ - the "drosophila system" of organic molecular beam epitaxy-with the slightly smaller but otherwise very similar molecule NTCDA. Indeed the adsorption properties, at first glance, are very similar: both molecules form highly ordered monolayers on suitable substrates, e.g., on $\operatorname{Ag}(111) ;^{20,21,23,24}$ both adsorb in a flat

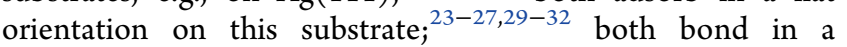
chemisorptive way to the substrate involving particularly the frontier orbitals which hybridize with the metallic s- and dstates including the surface states; ${ }^{29-38}$ in both cases, the first two layers seem to grow layer by layer and then continue to grow in crystallites in a Stranski-Krastanov fashion; ${ }^{11-13}$ both molecules apparently form molecular crystals under proper conditions.

However, a closer look reveals major differences in their adsorption behavior which cannot be understood by simple "extrapolation". NTCDA turns out to be much more complicated. Already in the monolayer more than three different superstructures exist depending on coverage. ${ }^{20-23}$ Even an upright standing molecule can be squeezed into the first layer at low temperatures. ${ }^{24}$ A reversible order-disorder phase transition is observed which goes in the "wrong" direction: the 2-dim long-range order of the monolayer disappears upon cooling the sample from room temperature to $150 \mathrm{~K}$ or below and returns after warming up with a small hysteresis. $^{27}$

In the present work, we try to understand those processes and results that occur in the mono/bilayer regime by direct observation of the dynamic development and its correlation to results known from the literature. The fortuitous advantage of the present study is that the five different observable states in this coverage range have significantly different work functions/ attenuation properties such that they can clearly be distinguished by UV-PEEM. This enabled the direct observation of the 2-dim gas phase state ("lattice gas") which is highly mobile, diffuses over large distances, and is apparently in equilibrium with the 2-dim islands of the r-phase in a certain temperature and coverage range.

The idea of a 2-dim gas phase or lattice gas is of course not new. Since the early days of surface science, the idea of highly mobile 2-dim gas phase states has been used to successfully describe the observed adsorption and desorption kinetics in many adsorbate studies of atoms or small molecules. Moreover, numerous theoretical models for adsorption or growth behavior are successfully based on the concept of a lattice gas. Very often the absence of an ordered phase in an experimental investigation and hence the invisibility of the deposited species by the used method is interpreted as occurrence of a 2-dim gas phase or lattice gas, and even pair correlation functions have been measured by STM and were taken as proof of this concept. ${ }^{61}$

In the present study, we measure the 2-dim gas phase or lattice gas directly and determine its density and homogeneity, 
and its competition with island formation. On the basis of the dynamic information from the movies and their quantitative evaluation we come to the following picture: The islands can only nucleate above a certain coverage of this lattice gas depending on temperature. For temperatures slightly below the temperature of desorption into the vacuum $(\sim 370 \mathrm{~K})$ this lattice gas can reach a coverage up to half a monolayer which is an extremely dense "gas". In contrast, for temperatures at and slightly below room temperature the density of the lattice gas is only a few percent of a monolayer, and nucleation of r-phase islands starts much earlier. The origin for this temperature behavior is most probably the lateral (intermolecular) bonding between molecules within an island. This lateral bonding arises either from the electrostatic quadrupole moments, or from van der Waals interaction, or through the substrate via the chemisorptive bond, or by a combination of these forces. Its magnitude is in the order of $0.2-0.3 \mathrm{eV}$ as derived from the Arrhenius plot of Figure 5, which is governed by the activation energy, $E_{\text {des }}$, of 2-dim desorption (desorption from the islands into the 2-dim gas phase). Thus, we have the following situation: for each (submonolayer) coverage there is a temperature below which island nucleation is found. In this case, desorption from the rims of the islands into the lattice gas and adsorption from the lattice gas at the islands are in equilibrium. For higher coverages more and larger islands are formed. For higher temperatures this equilibrium is shifted toward the 2-dim gas phase since the rate of desorption from the islands is proportional to $\exp \left(-E_{\text {des }} / k T\right)$ while the rate of adsorption is only weakly temperature dependent.

The observation of "jumping islands" which reminds of the motion of a water droplet on a hot plate is most likely also connected to the lattice gas because it also suggests that the molecules are very mobile on the surface and that they can easily be moved or shifted. The interesting observation is that they do it in a coordinated way, at least on the time scale of seconds. A droplet of a million molecules can thus "jump" by a micrometer distance back and forth. Of course, we do not postulate that the motion of the involved molecules is coherent, i.e., that these molecules move together as an assembly. They "desorb" quasi simultaneously from the island into the rather dense 2-dim gas phase which is then locally oversaturated such that a similar number of molecules must condense somewhere else. It is surprising that this condensation can happen at a micrometer distance from the location of "evaporation", and that this process of evaporation/ condensation involves a million of molecules simultaneously (within the time scale of few seconds). We speculate that collective phenomena such as density fluctuations of the lattice gas may play an important role. In any case, this observation supports our finding that the molecules are extremely mobile in lateral direction although they prefer a well-defined (LEED, STM results!) position and experience a significant chemical bonding to the substrate. Thus, we conclude that the lateral corrugation of the vertical potential (bonding) is very small although the magnitude of the vertical potential must be significant and is site-specific as indicated by the commensurate superstructure of the r-phase. Such a small lateral corrugation would also explain why additional molecules can be squeezed into the r-phase in order to form the c-phase which is also highly ordered but incommensurate. Thus, the energy loss of a less favorable adsorption site in the c-phase is overcompensated by the energy gain from more molecules.
A small lateral corrugation would also explain why several highly ordered superstructures were observed by LEED between $\mathrm{r}$ - and c-phase ${ }^{22,28}$ and why under certain preparation conditions needle-like c-phases develop (see Figure 7). It is therefore no surprise that the needle-like c-phase structures look different in different experiments and that they only occur under certain preparation conditions. Under these circumstances, it is also understandable that adsorption and desorption processes are completely reversible in the mono/ bilayer range because they occur close to (lateral) equilibrium, although this may be rather different for other organic molecules as shown most recently for PTCDA/Au(111). ${ }^{18}$ And finally the "strange" phase transition mentioned above ${ }^{27}$ appears much more plausible because a small parameter change, for instance a different expansion of substrate and adsorbate layer upon temperature change, may easily change the optimum adsorption site and hence the chemical interaction, if the lateral corrugation of the vertical potential is very small.

Such detailed spatially resolved dynamical observations as presented here are only possible if one can use a spectromicroscope like the SMART with its complementary analysis methods, which can be adapted to the questions that need to be answered.

\section{ASSOCIATED CONTENT}

\section{S Supporting Information}

The Supporting Information is available free of charge on the ACS Publications website at DOI: 10.1021/acs.jpcc.8b08523.

Movie 1 is a supplement to Figure 1 and shows the growth of NTCDA on the $\operatorname{Ag}(111)$ surface at $350 \mathrm{~K}$, as observed in UV-PEEM, with the field-of-view (FoV) the same as in the figure (AVI)

Movie 2 is a supplement to Figure 2 and shows the growth of NTCDA on the $\operatorname{Ag}(111)$ surface at $283 \mathrm{~K}$, as observed in UV-PEEM, with the FoV the same as in the figure (AVI)

Movie 3 is a supplement to Figure $6 a-d$ and shows the mesoscopic island mobility during growth of NTCDA on the $\operatorname{Ag}(111)$ surface at $350 \mathrm{~K}$, as observed in UVPEEM (AVI)

Movie 4 is a supplement to Figure $6 \mathrm{e}-\mathrm{h}$ and shows the mesoscopic island mobility during growth of NTCDA on the $\operatorname{Ag}(111)$ surface at $283 \mathrm{~K}$, as observed in UVPEEM (AVI)

\section{AUTHOR INFORMATION}

\section{Corresponding Author}

*(T.S.) E-mail: schmidtt@fhi-berlin.mpg.de.

ORCID

Thomas Schmidt: 0000-0003-4389-2080

Rainer H. Fink: 0000-0002-6896-4266

\section{Notes}

The authors declare no competing financial interest.

\section{ACKNOWLEDGMENTS}

The authors like to cordially thank Prof. H.-J. Freund for enabling experiments with the SMART instrument and for supporting the further development of this unique instrument. Financial support by the Federal German Ministry of Education and Research (BMBF) under Contracts 05 
KS4WWB/4 and 05K16WED (R.H.F.) as well as by the MaxPlanck Society is gratefully acknowledged. We thank the Helmholtz-Center Berlin for Materials and Energy for the allocation of synchrotron radiation beamtime and the BESSYII staff, especially Matthias Mast, Fred Senf, and Christian Jung, for technical support.

\section{REFERENCES}

(1) Leo, K. Organic photovoltaics. Nature Reviews Materials 2016, 1 (8), 16056.

(2) Hedley, G. J.; Ruseckas, A.; Samuel, I. D. Light harvesting for organic photovoltaics. Chem. Rev. 2017, 117 (2), 796-837.

(3) Muccini, M. A bright future for organic field-effect transistors. Nat. Mater. 2006, 5 (8), 605-13.

(4) Organic light-emitting diodes (OLEDs), materials, devices and applications; Woodhead Publishing Limited: Oxford, Cambridge, U.K., Philadelphia, PA, and New Delhi, India, 2013.

(5) Choi, S.; Kwon, S.; Kim, H.; Kim, W.; Kwon, J. H.; Lim, M. S.; Lee, H. S.; Choi, K. C. Highly flexible and efficient fabric-based organic light-emitting eevices for clothing-shaped wearable displays. Sci. Rep. 2017, 7 (1), 6424.

(6) Cahen, D.; Kahn, A.; Umbach, E. Energetics of molecular interfaces. Mater. Today 2005, 8 (7), 32-41.

(7) Otero, R.; Vázquez de Parga, A. L.; Gallego, J. M. Electronic, structural and chemical effects of charge-transfer at organic/inorganic interfaces. Surf. Sci. Rep. 2017, 72 (3), 105-145.

(8) Gebauer, W.; Langner, A.; Schneider, M.; Sokolowski, M.; Umbach, E. Exciton dispersion in a $\pi$-conjugated oligomer film: $\alpha$ quaterthiophene on highly oriented pyrolytic graphite and $\mathrm{Ag}(111)$. Phys. Rev. B: Condens. Matter Mater. Phys. 2004, 69 (12), 125420.

(9) Gebauer, W.; Langner, A.; Schneider, M.; Sokolowski, M.; Umbach, E. Luminescence quenching of ordered $\pi$-conjugated molecules near a metal surface: Quaterthiophene and PTCDA on $\mathrm{Ag}(111)$. Phys. Rev. B: Condens. Matter Mater. Phys. 2004, 69 (15), 155431.

(10) Schneider, M.; Umbach, E.; Sokolowski, M. Growth-dependent optical properties of 3,4,9,10-perylenetetracarboxylicacid-dianhydride (PTCDA) films on $\operatorname{Ag}(111)$. Chem. Phys. 2006, 325 (1), 185-192.

(11) Kilian, L.; Umbach, E.; Sokolowski, M. Molecular beam epitaxy of organic films investigated by high resolution low energy electron diffraction (SPA-LEED): 3,4,9,10-perylenetetracarboxylicacid-dianhydride (PTCDA) on Ag(111). Surf. Sci. 2004, 573 (3), 359-378.

(12) Marchetto, H.; Schmidt, T.; Groh, U.; Maier, F. C.; Levésque, P. L.; Fink, R. H.; Freund, H. J.; Umbach, E. Direct observation of epitaxial organic film growth: temperature-dependent growth mechanisms and metastability. Phys. Chem. Chem. Phys. 2015, 17 (43), 29150-60.

(13) Marchetto, H.; Groh, U.; Schmidt, T.; Fink, R.; Freund, H. J.; Umbach, E. Influence of substrate morphology on organic layer growth: PTCDA on $\operatorname{Ag}(111)$. Chem. Phys. 2006, 325 (1), 178-184.

(14) Lévesque, P. L.; Marchetto, H.; Schmidt, T.; Maier, F. C.; Freund, H. J.; Umbach, E. Correlation between substrate morphology and the initial stages of epitaxial organic growth: PTCDA/ $\operatorname{Ag}(111) . J$. Phys. Chem. C 2016, 120 (34), 19271-19279.

(15) Schmitt, S.; Schöll, A.; Umbach, E. Long-range surface faceting induced by chemisorption of PTCDA on stepped $\mathrm{Ag}(111)$ surfaces. Surf. Sci. 2016, 643, 59-64.

(16) Pollinger, F.; Schmitt, S.; Sander, D.; Tian, Z.; Kirschner, J.; Vrdoljak, P.; Stadler, C.; Maier, F.; Marchetto, H.; Schmidt, T.; Schöll, A.; Umbach, E. Nanoscale patterning, macroscopic reconstruction, and enhanced surface stress by organic adsorption on vicinal surfaces. New J. Phys. 2017, 19 (1), 013019.

(17) Schmitt, S.; Schöll, A.; Umbach, E. Multitude of PTCDA superstructures on $\operatorname{Ag}(111)$ and vicinal surfaces. J. Phys. Chem. C 2017, 121 (18), 9860-9868.

(18) Schmidt, T.; Marchetto, H.; Groh, U.; Fink, R. H.; Freund, H. J.; Umbach, E. Influence of substrate bonding and surface morphology on dynamic organic layer growth: perylenetetracarboxylic dianhydride on $\mathrm{Au}(111)$. Langmuir 2018, 34 (19), 5444-5453.

(19) Glöckler, K.; Seidel, C.; Soukopp, A.; Sokolowski, M.; Umbach, E.; Bohringer, M.; Berndt, R.; Schneider, W. D. Highly ordered structures and submolecular scanning tunnelling microscopy contrast of PTCDA and DM-PBDCI monolayers on $\mathrm{Ag}(111)$ and $\mathrm{Ag}(110)$. Surf. Sci. 1998, 405 (1), 1-20.

(20) Fink, R.; Gador, D.; Stahl, U.; Zou, Y.; Umbach, E. Substratedependent lateral order in naphthalene-tetracarboxylic-dianhydride monolayers. Phys. Rev. B: Condens. Matter Mater. Phys. 1999, 60 (4), $2818-2826$

(21) Stahl, U.; Gador, D.; Soukopp, A.; Fink, R.; Umbach, E. Coverage-dependent superstructures in chemisorbed NTCDA monolayers: a combined LEED and STM study. Surf. Sci. 1998, 414 (3), 423-434.

(22) Kilian, L.; Stahl, U.; Kossev, I.; Sokolowski, M.; Fink, R.; Umbach, E. The commensurate-to-incommensurate phase transition of an organic monolayer: A high resolution LEED analysis of the superstructures of NTCDA on $\operatorname{Ag}(111)$. Surf. Sci. 2008, 602 (14), 2427-2434.

(23) Braatz, C. R.; Ohl, G.; Jakob, P. Vibrational properties of the compressed and the relaxed 1,4,5,8-naphthalene-tetracarboxylic dianhydride monolayer on $\operatorname{Ag}(111)$. J. Chem. Phys. 2012, 136 (13), 134706.

(24) Braatz, C. R.; Esat, T.; Wagner, C.; Temirov, R.; Tautz, F. S.; Jakob, P. Switching orientation of adsorbed molecules: Reverse domino on a metal surface. Surf. Sci. 2016, 643, 98-107.

(25) Gador, D.; Buchberger, C.; Fink, R.; Umbach, E. "Manipulation" of molecular orientation in ultrathin organic films: NTCDA on $\mathrm{Ag}(111)$. Europhys. Lett. 1998, 41 (2), 231-236.

(26) Gador, D.; Buchberger, C.; Fink, R.; Umbach, E. Characterization of high-quality NTCDA films on metal substrates. J. Electron Spectrosc. Relat. Phenom. 1998, 96 (1-3), 11-17.

(27) Schöll, A.; Kilian, L.; Zou, Y.; Ziroff, J.; Hame, S.; Reinert, F.; Umbach, E.; Fink, R. H. Disordering of an organic overlayer on a metal surface upon cooling. Science 2010, 329 (5989), 303-5.

(28) Kilian, L. Adsorption, Struktur und Morphologie hochgeordneter organischer Adsorbatschichten. Ph.D. thesis, Universität Würzburg: Aachen, 2002.

(29) Stadler, C.; Hansen, S.; Schöll, A.; Lee, T. L.; Zegenhagen, J.; Kumpf, C.; Umbach, E. Molecular distortion of NTCDA upon adsorption on $\operatorname{Ag}(111)$ : a normal incidence $\mathrm{x}$-ray standing wave study. New J. Phys. 2007, 9, 50.

(30) Gador, D.; Zou, Y.; Buchberger, C.; Bertram, M.; Fink, R.; Umbach, E. NEXAFS investigations of NTCDA monolayers on different metal substrates. J. Electron Spectrosc. Relat. Phenom. 1999, $101,523-528$.

(31) Schöll, A.; Zou, Y.; Schmidt, T.; Fink, R.; Umbach, E. Highresolution photoemission study of different NTCDA monolayers on $\mathrm{Ag}(111)$ : Bonding and screening influences on the line shapes. J. Phys. Chem. B 2004, 108 (38), 14741-14748.

(32) Ziroff, J.; Hame, S.; Kochler, M.; Bendounan, A.; Scholl, A.; Reinert, F. Low-energy scale excitations in the spectral function of organic monolayer systems. Phys. Rev. B: Condens. Matter Mater. Phys. 2012, 85 (16), 161404.

(33) Marks, M.; Schöll, A.; Hofer, U. Formation of metal-organic interface states studied with 2PPE. J. Electron Spectrosc. Relat. Phenom. 2014, 195, 263-271.

(34) Zaitsev, N. L.; Nechaev, I. A.; Echenique, P. M.; Chulkov, E. V. Transformation of the $\operatorname{Ag}(111)$ surface state due to molecule-surface interaction with ordered organic molecular monolayers. Phys. Rev. B: Condens. Matter Mater. Phys. 2012, 85 (11), 115301.

(35) Tsirkin, S. S.; Zaitsev, N. L.; Nechaev, I. A.; Tonner, R.; Hofer, U.; Chulkov, E. V. Inelastic decay of electrons in Shockley-type metalorganic interface states. Phys. Rev. B: Condens. Matter Mater. Phys. 2015, 92 (23), 235434.

(36) Jakob, P.; Zaitsev, N. L.; Namgalies, A.; Tonner, R.; Nechaev, I. A.; Tautz, F. S.; Hofer, U.; Sanchez-Portal, D. Adsorption geometry and interface states: Relaxed and compressed phases of NTCDA/ 
Ag(111). Phys. Rev. B: Condens. Matter Mater. Phys. 2016, 94 (12), 125436.

(37) Rosenow, P.; Jakob, P.; Tonner, R. Electron-vibron coupling at metal-organic interfaces from theory and experiment. J. Phys. Chem. Lett. 2016, 7 (8), 1422-7.

(38) Tonner, R.; Rosenow, P.; Jakob, P. Molecular structure and vibrations of NTCDA monolayers on $\mathrm{Ag}(111)$ from densityfunctional theory and infrared absorption spectroscopy. Phys. Chem. Chem. Phys. 2016, 18 (8), 6316-28.

(39) Fink, R.; Weiss, M. R.; Umbach, E.; Preikszas, D.; Rose, H.; Spehr, R.; Hartel, P.; Engel, W.; Degenhardt, R.; Wichtendahl, R.; Kuhlenbeck, H.; Erlebach, W.; Ihmann, K.; Schlögl, R.; Freund, H. J.; Bradshaw, A. M.; Lilienkamp, G.; Schmidt, T.; Bauer, E.; Benner, G. SMART: A planned ultrahigh-resolution spectromicroscope for BESSY II. J. Electron Spectrosc. Relat. Phenom. 1997, 84 (1-3), 231-250.

(40) Wichtendahl, R.; Fink, R.; Kuhlenbeck, H.; Preikszas, D.; Rose, H.; Spehr, R.; Hartel, P.; Engel, W.; Schlögl, R.; Freund, H. J.; Bradshaw, A. M.; Lilienkamp, G.; Schmidt, T.; Bauer, E.; Benner, G.; Umbach, E. SMART: An aberration-corrected XPEEM/LEEM with energy filter. Surf. Rev. Lett. 1998, 05 (06), 1249-1256.

(41) Schmidt, T.; Groh, U.; Fink, R.; Umbach, E.; Schaff, O.; Engel, W.; Richter, B.; Kuhlenbeck, H.; Schlögl, R.; Freund, H.-J.; et al. XPEEM with energy-filtering: Advantages and first results from the smart project. Surf. Rev. Lett. 2002, 09 (01), 223-232.

(42) Rose, H.; Preikszas, D. Outline of a versatile corrected LEEM. Optik 1992, 92 (1), 31-44.

(43) Rose, H.; Preikszas, D. Time-dependent perturbation formalism for calculating the aberrations of systems with large ray gradients. Nucl. Instrum. Methods Phys. Res., Sect. A 1995, 363 (1-2), 301-315.

(44) Preikszas, D.; Rose, H. Correction properties of electron mirrors. J. Electron Microsc. 1997, 46 (1), 1-9.

(45) Schmidt, T.; Marchetto, H.; Lévesque, P. L.; Groh, U.; Maier, F.; Preikszas, D.; Hartel, P.; Spehr, R.; Lilienkamp, G.; Engel, W.; Fink, R.; Bauer, E.; Rose, H.; Umbach, E.; Freund, H. J. Double aberration correction in a low-energy electron microscope. Ultramicroscopy 2010, 110 (11), 1358-61.

(46) Schmidt, T.; Sala, A.; Marchetto, H.; Umbach, E.; Freund, H. J. First experimental proof for aberration correction in XPEEM: resolution, transmission enhancement, and limitation by space charge effects. Ultramicroscopy 2013, 126, 23-32.

(47) Weiss, M. R.; Follath, R.; Sawhney, K. J. S.; Senf, F.; Bahrdt, J.; Frentrup, W.; Gaupp, A.; Sasaki, S.; Scheer, M.; Mertins, H. C.; Abramsohn, D.; Schafers, F.; Kuch, W.; Mahler, W. The elliptically polarized undulator beamlines at BESSY II. Nucl. Instrum. Methods Phys. Res., Sect. A 2001, 467, 449-452.

(48) Bauer, E. Low energy electron microscopy. Rep. Prog. Phys. 1994, 57 (9), 895-938.

(49) Jia, J. J.; Esaulov, V. A.; Bendounan, A. Adsorption and desorption kinetics of NTCDA molecules on $\mathrm{Ag}(111)$ and $\mathrm{Au}(111)$ surfaces studied by ion scattering. Radiat. Eff. Defects Solids 2017, 172 (1-2), 39-47.

(50) Venables, J. A.; Spiller, G. D. T.; Hanbucken, M. Nucleation and Growth of Thin-Films. Rep. Prog. Phys. 1984, 47 (4), 399-459.

(51) Brune, H.; Bales, G. S.; Jacobsen, J.; Boragno, C.; Kern, K. Measuring surface diffusion from nucleation island densities. Phys. Rev. B: Condens. Matter Mater. Phys. 1999, 60 (8), 5991-6006.

(52) Chkoda, L.; Schneider, M.; Shklover, V.; Kilian, L.; Sokolowski, M.; Heske, C.; Umbach, E. Temperature-dependent morphology and structure of ordered 3,4,9,10-perylene-tetracarboxylicacid-dianhydride (PTCDA) thin films on $\mathrm{Ag}(111)$. Chem. Phys. Lett. 2003, 371 (5-6), $548-552$.

(53) Stöhr, J. NEXAFS spectroscopy. Springer Berlin Heidelberg: Berlin and Heidelberg, Germany, 1992.

(54) Schöll, A.; Fink, R.; Umbach, E.; Mitchell, G. E.; Urquhart, S. G.; Ade, H. Towards a detailed understanding of the NEXAFS spectra of bulk polyethylene copolymers and related alkanes. Chem. Phys. Lett. 2003, 370 (5-6), 834-841.
(55) Schöll, A.; Hübner, D.; Schmidt, T.; Urquhart, S. G.; Fink, R.; Umbach, E. Anharmonicity of the core-excited state potential of an organic molecule from NEXAFS vibronic fine structure. Chem. Phys. Lett. 2004, 392 (4-6), 297-302.

(56) Schöll, A.; Zou, Y.; Kilian, L.; Hübner, D.; Gador, D.; Jung, C.; Urquhart, S. G.; Schmidt, T.; Fink, R.; Umbach, E. Electron-vibron coupling in high-resolution X-Ray absorption spectra of organic materials: NTCDA on $\operatorname{Ag}(111)$. Phys. Rev. Lett. 2004, 93 (14), 146406.

(57) Schöll, A.; Zou, Y.; Hübner, D.; Urquhart, S. G.; Schmidt, T.; Fink, R.; Umbach, E. A comparison of fine structures in highresolution $\mathrm{x}$-ray-absorption spectra of various condensed organic molecules. J. Chem. Phys. 2005, 123 (4), No. 044509.

(58) Zou, Y.; Kilian, L.; Schöll, A.; Schmidt, T.; Fink, R.; Umbach, E. Chemical bonding of PTCDA on Ag surfaces and the formation of interface states. Surf. Sci. 2006, 600 (6), 1240-1251.

(59) Scholz, M.; Sauer, C.; Wiessner, M.; Nguyen, N.; Scholl, A.; Reinert, F. Structure formation in organic thin films observed in real time by energy dispersive near-edge $\mathrm{x}$-ray absorption fine-structure spectroscopy. New J. Phys. 2013, 15, 083052.

(60) Taborski, J.; Väterlein, P.; Dietz, H.; Zimmermann, U.; Umbach, E. NEXAFS investigations on ordered adsorbate layers of large aromatic molecules. J. Electron Spectrosc. Relat. Phenom. 1995, $75,129-147$.

(61) Matvija, P.; Rozboril, F.; Sobotik, P.; Ost'adal, I.; Kocan, P. Pair correlation function of a $2 \mathrm{D}$ molecular gas directly visualized by scanning tunneling microscopy. J. Phys. Chem. Lett. 2017, 8 (17), $4268-4272$ 